\title{
Thoracic epidural block - A sole anasthetic procedure for breast surgeries
}

\author{
Durga Shankar Patel ${ }^{1}$, Omprakash Sundrani ${ }^{2, *}$, Sanjay Goyal $^{3}$, A. M. Lakara ${ }^{4}$ \\ ${ }^{\mathbf{1}}$ Associate Professor, ${ }^{\mathbf{2}}$ Assistant Professor, ${ }^{3}$ Director and Consultant, ${ }^{4}$ Professor and HOD, ${ }^{1,3,4}$ Dept. of Anaesthesiology, ${ }^{2}$ Dept. \\ of Anaesthesiology and Critical Care, ${ }^{1}$ Government Medical College Ambikapur, Chhattisgarh, ${ }^{2}$ Pt. Jawahar Lal Nehru Memorial \\ Medical College, Raipur, Chhattisgarh, ${ }^{3}$ Sparsh Multispecialty Hospital, Bhilai Chhattisgarh, ${ }^{4}$ Late Shri Lakhiram Agrawal \\ Memorial Government Medical College, Raigarh, Chhattisgarh, India
}

\section{*Corresponding Author: Omprakash Sundrani}

Email: sundraniop@ rediffmail.com

Received: $24^{\text {th }}$ February, 2018

Accepted: $14^{\text {th }}$ July, 2018

\begin{abstract}
Introduction: Breast cancer is one of the most common cancers in middle and old aged females. Oncological breast surgeries have commonly been performed under General Anaesthesia (GA). Thoracic Epidural Anaesthesia (TEA) is a safe and advantageous technique associated with fewer post surgical recovery complications, shorter hospital stay and consequently, decreased health care costs. TEA provides better control of pain and provides satisfactory analgesia in postoperative period without any respiratory complications.

Materials and Methods: A prospective study was carried out in Anaesthesiology and Critical care department of Pt. J.N.M. Medical College and Dr. B.R.A.M. Hospital, Raipur (C.G.) in 40 female patients aged between 30 and 60 years with ASA grade I, II, III underwent for breast surgeries during February 2007 to July 2008. Pre operative assessment of the patient including history, clinical examination and relevant investigations were done. Pre operative assessment of the patient including history, clinical examination and relevant investigations were done. Explanation regarding procedure and consent was taken from all the patients. The procedure and techniques were performed as per standard protocol.

Observations: Maximum numbers of cases (32 cases, 80\%) were in the age group of 45-60 yrs. Mean age in our study was 49.0 \pm 6.6 yrs. The mean duration of onset of block was $7.67 \pm 1.14 \mathrm{~min}$. The mean duration was $117.12 \pm 28.90 \mathrm{~min}$. In maximum number of patients (21), duration of surgery was 91-120 min and mean duration of surgery was 123.1. $\pm 13.16 \mathrm{~min}$.Preoperative mean SBP was $120 \pm 7.45 \mathrm{~mm}$ of $\mathrm{Hg}$. Intraoperative SBP ranged from $119.8 \pm 7.02 \mathrm{~mm}$ of $\mathrm{Hg}$ to $125.15 \pm 8.43 \mathrm{~mm}$ of $\mathrm{Hg}$. Preoperative mean DBP was $77.6 \pm 4.34 \mathrm{~mm}$ of $\mathrm{Hg}$. Introperative DBP ranged from $71 . \pm 5.61 \mathrm{~mm}$ of $\mathrm{Hg}$ to 77 . $\pm 4.63 \mathrm{~mm}$ of $\mathrm{Hg}$. Preoperative mean of PR was $84.1 \pm 5.12 / \mathrm{min}$. Intraoperative mean PR ranged from $80.4 \pm 4.41 / \mathrm{min}$ to $83.35 \pm 4.54 / \mathrm{min}$ and minimum PR was $80.4 \pm 4.41 / \mathrm{min}$. Preoperative mean of RR was $14.52 \pm 1.3 / \mathrm{min}$. It ranged between $13.35 \pm 0.97 / \mathrm{min}$ to 14.27 $\pm 0.93 / \mathrm{min}$.
\end{abstract}

Conclusion: It is concluded that TEA for breast surgeries is a safe and a reliable alternative of GA.

Keywords: Thoracic epidural block, Breast cancer surgery.

\section{Introduction}

Breast cancer is one of the most common cancers in middle and old aged females. Breast surgeries are the common surgical procedure for breast cancer. Oncological breast surgeries have commonly been performed under General Anaesthesia (GA). GA does not eliminate the surgical stress response, may cause undesirable side effects, and cannot provide postoperative pain control. The routine use of parenteral opioids is still inadequate for post operative pain control and may further aggravate the unpleasant side effects such as nausea, vomiting, sedation, impaired oxygenation and depressed ventilation. Regional anaesthesia is advantageous in more than one way like less sedation, reduced intraoperative blood loss, reduced postoperative catabolism, reduced thromboembolism, reduced nausea and vomiting, improved pulmonary function, early ambulation, decreased time for hospital stay, cost effectiveness, better post operative pain control and improved ventilatory function. ${ }^{1-3}$

Various regional anaesthetic technique for breast surgeries have been suggested including -Thoracic epidural block, cervical epidural block, Local anaesthetic infiltration, Field block, Intercostal nerve block, Paravertebral block, Brachial plexus block have been used either as an alternative or as an adjuvant to GA.

However because of technical difficulties, inherent limitations of anaesthetic and analgesic effects, and increased possibilities of complications, the applications of some of these approaches are not entirely satisfactory in anaesthetic management of extensive breast surgeries and in postoperative pain control. $^{4}$

TEA is a safe and advantageous technique associated with fewer post surgical recovery complications, shorter hospital stay and consequently, decreased health care costs. Furthermore Epidural Anaesthesia (EA) and analgesia have been associated with a reduction in the incidence and severity of much perioperative physiological trepidations. Thoracic epidural anaesthesia selectively blocks cardiac sympathetic fibers and this offers potential benefits like attenuation of surgical stress response, improvement of 
myocardial oxygen balance, and stabilization of intraoperative hemodynamic. ${ }^{5,6}$

It should be emphasized that recurrence of malignancies is contributed by several factors like immunosuppression because of stress response during general anesthesia. It has been observed that incidence of recurrence of malignancies has been greatly reduced by thoracic epidural anaesthesia. ${ }^{7,8}$

High-risk surgical patient of breast cancer who received epidural anaesthesia had a reduction in over all post operative complication rates in the form of cardiovascular failure, respiratory failure and major infectious complications. Thoracic epidural block is globally acceptable alternative to general anesthesia in high-risk surgical patients. ${ }^{1,9}$

TEA provides better control of pain and provides satisfactory analgesia in postoperative period without any respiratory complications. ${ }^{10}$

Keeping in mind, various advantages of thoracic epidural technique and limitations in GA, this study was carried out to evaluate feasibility, effectiveness and potential complications of TEA as a sole anaesthetic technique.

\section{Materials and Methods}

A prospective study was carried out in Anaesthesiology and Critical care department of Pt. J.N.M. Medical College and Dr. B.R.A.M. Hospital, Raipur (C.G.) in 40 female patients aged between 30 and 60 years with ASA grade I, II, III underwent for breast surgeries during February 2007 to July 2008.

Exclusion Criteria: History of allergic reaction to local anaesthestic, bleeding diathesis, anticoagulant therapy or prolonged aspirin ingestion, local and systemic infection, psychiatric illness, chronic headache and backache in the past.

Pre operative assessment of the patient including history, clinical examination and relevant investigations were done. Explanation regarding procedure and consent was taken from all the patients.

The procedure and techniques were performed as per standard protocol. ${ }^{11}$

In sitting position, under all the aseptic precautions, T2-T3 or T3-T4 was identified by palpating downwards from C7 vertebra and local infiltration of $2 \mathrm{ml} 2 \%$ Lignocaine was done at the level of T2-T3 or T3-T4 interspaces. An incision with 15 number blade was made to facilitate the entry of Tuohy epidural needle. An epidural needle 16-18G was inserted through the midline approach and epidural space was found by Loss of resistance method. A disposable sterile multihole 16 $-18 \mathrm{G}$ epidural catheter 2-3 cm cephalad was threaded in the epidural space and was secured with adhesive. The patient was placed in supine position. A test dose (Moore et al, 1995) ${ }^{11}$ of $2 \mathrm{ml}$ 2\% Lignocaine with Adr. (1: 200000) was given to exclude the possibility of intradural or intravascular placement of catheter. After 10 minutes with negative test dose confirmation $10 \mathrm{ml}$ of $0.375 \%$ Bupivacaine plain (which was made by adding $2.5 \mathrm{ml}$ normal saline in $7.5 \mathrm{ml} 0.5 \%$ Bupivacaine) and Fentanyl $25 \mu \mathrm{gm}$ was given through epidural catheter. Onset of block and extent of block was assessed.

Intraoperative sedation was given with inj. Midazolam IV $0.03 \mathrm{mg} / \mathrm{kg}$ and in addition to that 50 $\mu \mathrm{gm} / \mathrm{kg} / \mathrm{min}$ propofol infusion was given if required. Oxygen supplement when needed was given via facemask $31 \mathrm{tr} / \mathrm{min}$ intra operatively. Intra operative top up doses was given with $5 \mathrm{ml} 0.375 \%$ Bupivacaine and duration of block was noted.

\section{Monitoring}

Intra operative monitoring was done for PR, SBP, $\mathrm{DBP}, \mathrm{MAP}, \mathrm{RR}$, and $\mathrm{SpO} 2$ initially at 5 minutes intervals for 20 minutes then after 10 minutes and subsequently at 15 minutes intervals. During the procedure patients were monitored for any complications and side effects and managed accordingly.

\section{Haemodynamic Changes}

1. PR below $60 / \mathrm{min}$ was considered as bradycardia.

2. SBP and DBP were considered as significant hypotension when fall in the B.P. was more than $20 \%$ of preoperative value.

\section{Respiratory Changes}

Fall in $\mathrm{SpO}_{2}$ below $90 \%$ and any respiratory distress was considered significant.

\section{Complications and Side Effects}

Throughout the procedure, we watched for any side effect and complications related to technique and anaesthetic drugs via thoracic epidural route.

The collected data were put in the master chart. The results were analyzed by various statically techniques like percentage, mean, standard deviation, paired $\mathrm{T}$ test and mean value with the help of MS Excel.

\section{Discussion}

Thoracic epidural technique provides selective blockade of the surgical site, with diminished requirements of opioids and local anaesthetics. In addition, it has recently been utilized in the management of acute and chronic pain syndromes, such as postoperative thoracotomy pain and pain due to cancer. In patients with coronary artery disease, TEA relieves anginal pain and provides early mobility and stabilization. ${ }^{12,13}$

The mean onset time of block was $7.67 \pm 1.14$ min in the current study, whereas the onset time was 3-8 min as observed by Stephen JH et al $(2001)^{14}$ while it was $7.05 \pm 1.5 \mathrm{~min}$ as observed by Swadia VN et al (2002). ${ }^{9}$

Bupivacaine and Fentanyl has little higher onset time as compared to Lidocaine or Ropivacaine. The late onset of Bupivacine was due to its different 
pharmokinetic profile ( $\mathrm{pH}, \mathrm{pKa}$, lipid solubility, fractional unionization) anesthetics.

Duration of analgesia was slightly lesser in Swadia VN et al (2002) $)^{9}$ than our study. Probably because they used combination of Lidocaine and Bupivacaine and we used Bupivacaine and Fentanyl and because of it and presence of Fentanyl we achieved longer duration of action. So duration of EA is principally affected by choice of local anaesthetic and presence and absence of adjuvant.

The duration of surgery in Groeben $\mathrm{H}$ et al $(2002)^{15}$ matched with our study.

In our study, 10 segments block achieved in $41.02 \%$ cases, 11 segments in $35.89 \%$ cases, 9 segments in $17.94 \%$ cases and 8 segments block in $5.13 \%$ cases with $2 \mathrm{ml}, 2 \%$ Lignocaine with Adr.(1:200,000) $+10 \mathrm{ml} \quad 0.375 \%$ Bupivacaine and $25 \mu \mathrm{gm}$ Fentanyl and catheter was placed between $\mathrm{T} 2$ to $\mathrm{T} 3$ or $\mathrm{T} 3$ to $\mathrm{T} 4$ interspaces.

Gruber EM et al $(2001)^{13}$-achieved 6 segments block from $\mathrm{T} 2$ to $\mathrm{T} 8$ with 10 to $12 \mathrm{ml}, 0.25 \%$ Bupivacaine. The catheter was placed at $\mathrm{T} 4$ to $\mathrm{T} 5$ interspaces. They need slightly higher drugs probably because they used lesser concentration of drug and catheter was placed little lower.

Swadia VN et al $(2002)^{9}$-achieved 7 to 8 segmental block from T1/ T2 to T8 with $1.5 \mathrm{ml} / \mathrm{segmental}$ drugs containing equal amount of $2 \%$ Lidocaine and 5\% Bupivacaine in $92 \%$ cases. They required more drug for each segment, probably because placement of the catheter was at lower level.

The principal factors affecting the spread of EA are dose (volume times concentration) and site of injection.

It is evident from different studies that after TEA, fall in BP occurred even with different local anaesthetic due to symphathectomy. Fall in BP occurred more with larger drugs and in older age groups, but it was in acceptable range in most of the cases after managing with simple measures. ${ }^{15-17}$

Epidural anaesthsia extending to high level (T1 to T4) leads to blockade of the preganglionic cardio accelerator fibers which results in reduction in PR.

Swadia et al $(2002)^{9}$ and Stephen JH et al $(2001)^{14}$ were found that Bradycardia may occur after any local anaesthetic given via thoracic epidural route and it is not because of the particular effect of any drug, in fact it is due to sympathetic blockade after TEA. The severity of bradycardia may vary with different drugs because of difference in their direct cardiac depression property.

Many studies showed that TEA improves ventillatory functions in many ways and it is a better option than GA even in COPD patients. Apart from other beneficial mechanism, Bupivacaine stimulates ventilatory functions due to direct stimulation after TEA. ${ }^{9,10,15}$
Few studies showed that mild side effects or complications may occur during breast surgery under TEA but they can be manageable easily. ${ }^{18,19}$

\section{Conclusion}

It is concluded that TEA using $10 \mathrm{ml}$ of $0.375 \%$ Bupivacaine and $25 \mu \mathrm{gm}$ Fentanyl for breast surgeries is a safe and a reliable alternative of GA. It can provide not only effective anaesthesia in terms of haemodynamic and respiratory stability but also leads to faster anaesthetic recovery and greater patient's satisfaction. TEA is associated with earlier hospital discharge and lower incidence of various complications. Thus, we can conclude that "Thoracic epidural Anaesthesia is feasible, safe, effective and economic procedure for breast surgeries. It provides early recovery and greater patient's satisfaction. So we strongly recommend it for breast surgeries".

\section{Conflict of Interest: None.}

\section{References}

1. Ochroch EA, Gottschalk A, Augostides J. Long-term pain and activity during recovery from major thoracotomy using thoracic epidural analgesia. Anesthesiology, 2002;97:1234-1244.

2. Vaughan RS. Pain relief after thoracotomy. Br J Anaesth, 2001;87:681-683.

3. Doss NW, Ipe J, Crimi T. Continuous thoracic epidural anesthesia with $0.2 \%$ ropivacaine versus general anesthesia for perioperative management of modified radical mastectomy. Anesth Analg. 2001;92:1552-1557.

4. Borgeat A, Ekatodramis G, Schenker C. Postoperative nausea and vomiting in regional anesthesia: a review. Anesthesiology, 2003;98:530-547.

5. Maharajan SK, Srestha BM. Epidural anaesthesia and analgesia. Kathmandu University Medical Journal. 2004;2(1):18-20.

6. Murali C, Jawali V, Murali V. Conscious off pump coronary artery bypass surgery in a patient with end stage renal failure thoracic epidural anaesthesia as the sole anaesthesic. J Anaesth Clin Pharmacol. 2005;21(2):21720.

7. Kozian, Alf, Schilling. Non analgesic effects of thoracic epidural anaesthesia. Current opinion in Anaesthesiology. 2005;18(1):29-34.

8. Sessler DI, Sharmgar B, Edward JM. Can regional analgesia reduce the risk of recurrence after breast cancer? Contemporary clinical trial. 2008;29(4):517-26.

9. Swadia VN, Patel M, Vasava JC. Thoracic epidural anaesthesia for carcinoma breast surgery in high risk patients. Journal of Anaesthesia Clinical Pharmacology. 2002;18(2):187-91.

10. Ahmed A Shorrab, Nabil A, Usama. Intermittent thoracic epidural administration of ropivacaine, fentanyl vs bupivacine, fentanyl after thoracotomy. Internet Journal of Anaesthesiology. 2007;13:1.

11. Moore CM, Cross MH Desborough JP. Hormonal effect of thoracic extradural analgesia for cardiac surgery. British Journal of Anaesthesia. 1995;75:387-93.

12. Blomberg S, Curelaru I, Emanuelsson H. Thoracic epidural anaesthesia in patients with unstable angina pectoris. European Heart Journal. 1989;10(5):437-44. 
13. Gruber EM, Tschernko EM, Kritzinger M. The effects of thoracic epidural analgesia with Bupivacaine $0.25 \%$ on ventilatory mechanics in patients with severe chronic obstructive pulmonary disease. Anaesth Analg. 2001;92:1015-19.

14. Stephen J, Holman, Richard R. What constitutes and effective but safe initial dose of lidocaine to test a thoracic epidural catheter? Anaesth Analg. 2001;93:74054.

15. Groeben H, Schafer, Beatrix C. Effects of high thoracic epidural anaesthesia and local anaesthetic on bronchial hyperreactivity. J Clin Monit Comput. 2002;96(3):53641.

16. Simon Misha J G, Bernadette J, Veerng. The effect of age on neural blockade and haemodynamic changes after epidural anaesthesia with ropivacaine. Anaesthes Analg. 2002;94:1325-30.

17. Stefano C, Mangia F, Stelian E. High thoracic epidural anaesthesia for cardiac surgery. Text Heart Inst $J$. 2006;33;148-53.
18. Karagoz HY, Kurtugolu, Bakkaloglu. Coronary artery bypasses grafting in awake patients: Three year's experience in 137 patients. Journal of Thoracic and Cardiovascular Surgery. 2002;125:1401-04.

19. Ryu HG, Bank JH, Lee CJ. The coiling length of thoracic epidural catheters the influence of epidural approach angle. British Journal of Anaesthesia. 2007;98(3):401-04.

How to cite this article: Patel $\mathrm{D} S$, Sundrani $\mathrm{O}$, Goyal S, Lakara A. M. Thoracic epidural block - A sole anesthetic procedure for breast surgeries. Indian $\mathrm{J}$ Clin Anaesth. 2018;5(4):469-472. 